\title{
Switching of Multiple Mobile Robots Formation Control Based on Leader-Follower
}

\author{
Run Zhou ${ }^{1, \text { a }}$, Wei Xiang ${ }^{1, \text { b,* }}$ \\ ${ }^{1}$ College of Electrical and Information Engineering, Southwest University for Nationalities, \\ Chengdu, Sichuan, China 610041 \\ a2872994057@qq.com, b3730544@qq.com, ${ }^{*}$ corresponding author
}

Keywords: Multi-Robot, Leader-Follower Algorithm, Formation Switch, Coordinated Path to Follow

\begin{abstract}
At first, this article summarizes several methods of the current multi-robot formation control and introduces the basic idea of formation control algorithm based on leader-follower. Then the control algorithm is introduced into switching control process (the forming of the initial formation, maintenance and switching) of underwater formation. It is the purpose of this paper that the queue can transform the shape of itself to adjust the current underwater environment in the process of advancing, and arrive the destination safely. Finally, That is tested on two different environments with barrier in the $2 \mathrm{D}$ simulation platform. The simulation results show that the method is monolithic, flexible and simple in calculation, in addition, it can realize arbitrary transformation of formation.
\end{abstract}

\section{Introduction}

Both the development of ocean resources and the military task all are inseparable from multiple mobile robots formation control and collaboration. Especially, multi-robot system has prominent advantages compared with single robot system about stability, reliability, availability, fault tolerance and so on. Therefore, the formation control of multiple robots system has become one of the most important issues in the field.

Formation control is that a group of robots form and keep a shape to adapt the constraint of the current environment (such as obstruction or physical limitation of space) in the process of going to the target area [1]. With the development of formation control technology in the field, many formation control methods were put forward, mainly 3 kinds: Leader-follower method [2], based on behavior method [3] and virtual structure method [4]. Among them, the leader-follower system mode selects an individual as a leader, responsible for leading the entire formation tracking reference path, the remaining individuals as followers, followers by maintaining a given distance and azimuth angular with leader to form the desired formation [5].

This article aims to put leader-follower formation control algorithm into underwater queue, and take into account the requirements and constraints of external physical environments, according to different environments switching different formations in the process of performing tasks. This method not only guarantees the consistency and integrity of the formation, but also is flexible and high on the feasible region is not required. It can be widely used in various areas of team.

\section{The Queue's Design Idea about Forming, Maintenance and Switching}

At first, the robot fishes are scattered in the field, then the desired initial formation is formed rapidly by determining the relative position of the leader and followers (the initial position of the leader has been given). Driven by program, the queue will be led by the leader to move toward the target area with the designed shape. But in the process forward, the queue will often encounter a variety of obstacles, to maintain the initial formation will not be able to pass the hazard successfully. At this point, the formation should change the current formation, adapted to the specific environmental constraints. It is shown in Fig. 1: 


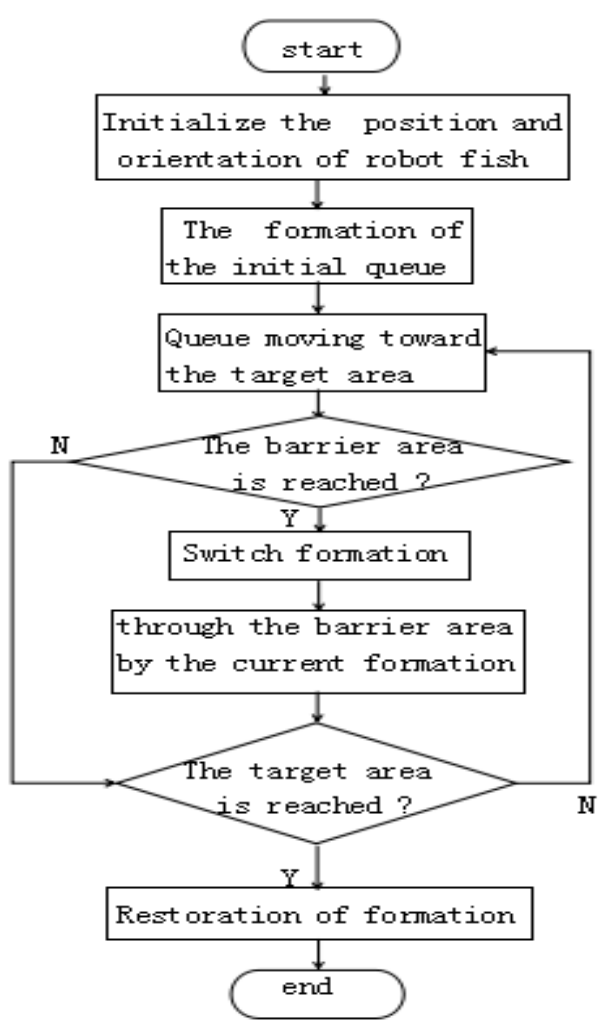

Fig. 1 The flowchart of queue in formation, maintenance and switching.

\section{Leader-Follower Algorithms for Formation Control Mechanisms}

To achieve the queue of the forming, maintenance and switching, real - time return the coordinates of the leader to follower. According to the formation relationship of specify location, follower constantly adjust itself position and direction, to meet the constraints of leader. In other words, the running path of the leader is the reference path of follower.

The core idea of the algorithm is that the leader of the current position coordinates is the reference point of follower input, but ignores the actual location of each robot fishes in the formation.

In this paper, a case study of two robot fishes to show the leader-follower formation control model, it is shown in Fig. 2:

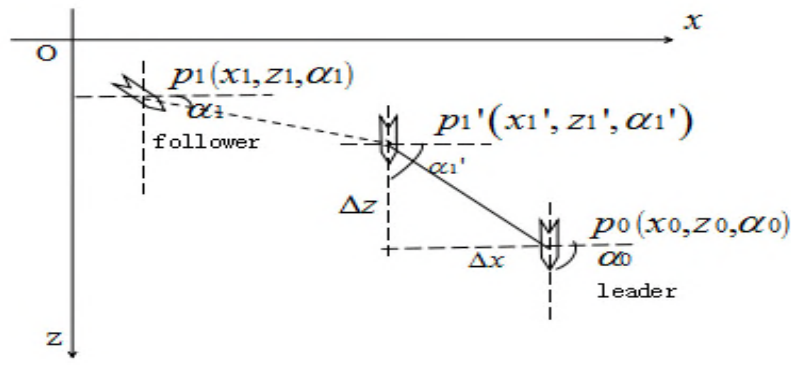

Fig. 2 Leader-follower formation control model.

The initial positions of leader and follower are $p_{0}\left(x_{0}, z_{0}, \alpha_{0}\right)$ and $p_{1}\left(x_{1}, z_{1}, \alpha_{1}\right)$, respectively. To form the specify formation $f=\left[\begin{array}{l}p_{0} \\ p_{1}{ }^{\prime}\end{array}\right]$, the follower target position should be $p_{1}{ }^{\prime}\left(x_{1}{ }^{\prime}, z_{1}{ }^{\prime}, \alpha_{1}{ }^{\prime}\right)$. Relative position relation can be expressed as $R_{i}$ : 


$$
\left\{\begin{array}{l}
x_{1}{ }^{\prime}=x_{0}-\Delta x \\
z_{1}{ }^{\prime}=z_{0}-\Delta z \\
\alpha_{1}{ }^{\prime}=\alpha_{0}-\Delta \alpha \quad \alpha_{i} \in(-\pi, 0] \cup(0, \pi]
\end{array}\right.
$$

This equation group set up communication model between leader and follower. When the queue reaches the barrier area, by changing $\Delta x, \Delta z, \Delta \alpha$ to switch formation. Different $\Delta x, \Delta z, \Delta \alpha$ for different formations, formation options can be set depending on the physical environment.

\section{Switching Process in Different Formations}

First, make the following definitions:

Custom 1: To better describe the idea of formation switching under the leader-follower control model, set the location of the obstacle are already known;

Custom 2: Each robot fish have a fixed number (for example: fish1, fish2, fish3...), and state of motion is stable in water;
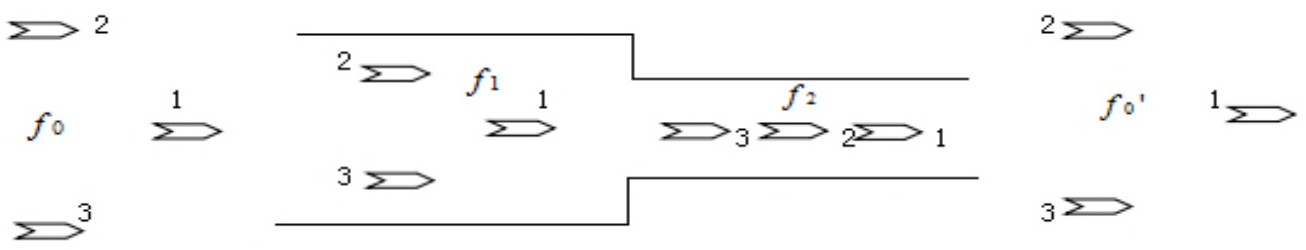

Fig. 3 The schematic diagram of barrier zone formation switch.

Custom 3: Setting the geometric center as coordinates center for each fish.

Leader-follower formation control model can realize arbitrary formation of transformation. This article only list 3 robot fishes, numbered respectively 1,2, 3 to show the two types of transformation processes of the three formations under the obstacles environment. It is shown in Fig. 3.

Fish1 is leader, and fish2, fish 3 all are followers. The formation is $f_{0}$ before reaching barrier zone. To adapt the obstacles environment, the formation should be switched as $f_{1}, f_{2}$ until through the barrier zone to recover formation $f_{0}{ }^{\prime}$

The Stage of Forming Initial Formation. At this stage, leader's initial position $p_{0}\left(x_{0}, z_{0}, \alpha_{0}\right)$ is already known, and target formation is $f_{0}$, so leader and followers relative location relationship is $R_{0}$. Followers according $p_{0}\left(x_{0}, z_{0}, \alpha_{0}\right)$ and $R_{0}$ automatically find itself desired location and toward in the formation $f_{0}$.

The Stage of Keeping Formation. After the initial queue $f_{0}$ finished, to accomplish the specified task, the queue will be led by the leader toward target area with keeping $f_{0}$ ( leader and follower relative location relationship $R_{0}$ unchanged, and to achieve consistency in formation forward, usually $\Delta \alpha=0$ ). Because the queue in the process of moving, leader position is constantly changing, the feedback of the reference point is dynamic changing. So the followers in order to meet the current rule $R_{0}$ of formation control, have to follow the leader's path and continually adjust themselves positions to achieve keeping and advance of queue.

The Stage of Switching Formation. When the queue reaches the barrier area, how to switch the formation to avoid barrier is the focus of this study. Transformation strategy is broken down as follows:

Relative transformation $f_{0} \stackrel{\text { switch }}{\longrightarrow} f_{1}$. Keep original geometric structure in formation, only to change the spacing between fishes size, compress queue space to achieve the avoidance. It is shown in Fig. 4. 


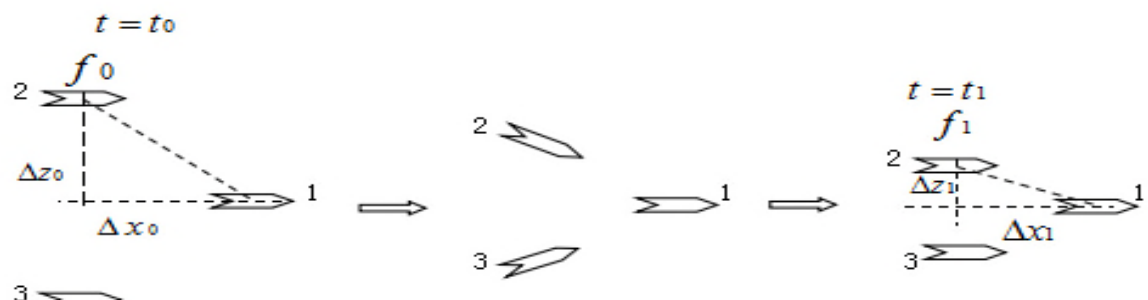

Fig. 4 Relative transformation $f_{0} \stackrel{\text { switch }}{\longrightarrow} f_{1}$

Step1: 1st fish ( leader ) does not participate the switching of formation, and set the current position is $p_{1}\left(x_{1}, z_{1}, \alpha_{1}\right)$. Changing the rule $R_{0}$ between follower2, follower3 and leader, makes $\Delta x_{0}, \Delta z_{0}$ respectively into $\Delta x_{1}, \Delta z_{1}$, and satisfies the inequalities $\left\{\begin{array}{l}\left|\Delta x_{1}\right|<\left|\Delta x_{0}\right| \\ \left|\Delta z_{1}\right|<\left|\Delta z_{0}\right|\end{array}\right.$. So we can build up the relationship of coordinate between leader and followers under the formation $f_{1}$ :

$$
\begin{aligned}
& \begin{cases}x_{2}=x_{1}-\Delta x_{1} & \Delta x_{1} \text { positive } \\
z_{2}=z_{1}-\Delta z_{1} & \Delta z_{1} \text { positive }\end{cases} \\
& \begin{cases}x_{3}=x_{1}-\Delta x_{1} & \Delta x_{1} \text { positive } \\
z_{3}=z_{1}-\Delta z_{1} & \Delta z_{1} \text { negative }\end{cases}
\end{aligned}
$$

The $\left(x_{1}-\Delta x_{1}, z_{1}-\Delta z_{1}\right)$ is expected position of follower2, follower3 in the formation $f_{1}$ at $t_{1}$ moment;

Step2: When follower2 and follower3 have reached $\left(x_{1}-\Delta x_{1}, z_{1}-\Delta z_{1}\right)$, restoring fish toward, ordering $\Delta \alpha_{1}=0,\left\{\begin{array}{l}\alpha_{2}=\alpha_{1} \\ \alpha_{3}=\alpha_{1}\end{array}\right.$ Complete the switch $f_{0} \stackrel{\text { switch }}{\longrightarrow} f_{1}$.

Absolute Transformation $f_{0} \stackrel{\text { switch }}{\longrightarrow} f_{1}$. When facing obstacles, as narrow path, formation to reduce the queue's space can not avoidance, it is needed to change absolutely for formation (queue's geometric structure and rules will be changed). It is shown in Fig. 5.

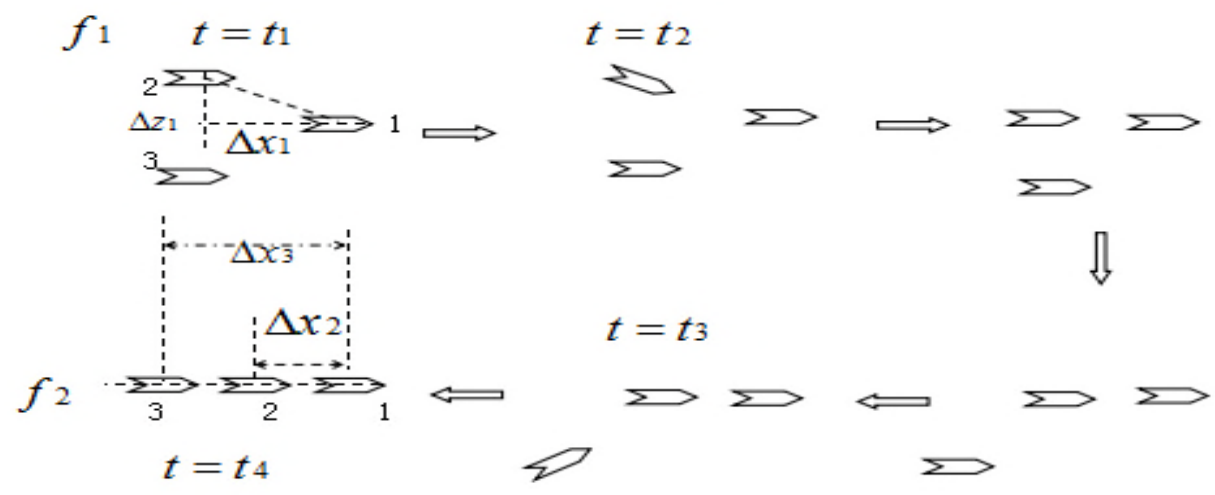

Fig. 5 Absolute transformation $f_{1} \stackrel{\text { switch }}{\longrightarrow} f_{2}$.

The strategy of a single follower position transformation is same as the former, not repeat it here. But here, it is needed to point out the order of the followers transform: $t_{2}$ moment, the follower2 coordinate relation becomes $\left\{\begin{array}{l}x_{2}=x_{1}-\Delta x_{2} \\ z_{2}=z_{1}\end{array}\right.$, while the follower3 remain $\left\{\begin{array}{l}x_{3}=x_{1}-\Delta x_{1} \\ z_{3}=z_{1}-\Delta z_{1}\end{array}\right.$;At $t_{3}$ 
moment, follower 2 has reached expected position in the formation $f_{2}$, and adjusted its toward to meet $\alpha_{2}=\alpha_{1}$. The follower2 coordinate is still $\left\{\begin{array}{l}x_{2}=x_{1}-\Delta x_{2} \\ z_{2}=z_{1}\end{array}\right.$, but follower3 changes into $\left\{\begin{array}{l}x_{3}=x_{1}-\Delta x_{3} \\ z_{3}=z_{1}\end{array}\right.$. According to the Fig. 5, we can see $\left|\Delta x_{3}\right|>\left|\Delta x_{2}\right|$, specific values depending on various circumstances. Finally, At $t_{4}$ moment, adjusts the point of follower 3 to meet $\alpha_{3}=\alpha_{1}$, so the switching of $f_{1} \stackrel{\text { switch }}{\longrightarrow} f_{2}$ is finished.

After the queue through the obstacles path, recovers the original formation $f_{0}{ }^{\prime}$ in the same strategy and continues to advance to the target area.

\section{Validation of Simulation Platform}

In order to verify the feasibility of formation control algorithm, the algorithm is tested in 2D simulation platform with the model Fig. 3 , information switching $f_{0}, f_{1}, f_{2}$. In the process, 4 robot fishes respectively numbered $6,7,8,9$ to build a simulation barrier environment, Similarly, the destination queue is consist of 3 robot fishes respectively numbered $2,3,4$. The relative rules of different formations are shown in the Table (1):

Table 1. The $(\Delta x, \Delta z, \Delta \alpha)$ value respectively in the formations of $f_{0}, f_{1}, f_{2}, f_{0}{ }^{\prime}$.

\begin{tabular}{|c|c|c|c|}
\hline formation & Fish1 coordinate & Fish2 coordinate & Fish3 coordinate \\
\hline$f_{0}$ & $(0,0,0)$ & $(500,650,0)$ & $(500,-650,0)$ \\
\hline$f_{1}$ & $(0,0,0)$ & $(400,400,0)$ & $(400,-400,0)$ \\
\hline$f_{2}$ & $(0,0,0)$ & $(500,0,0)$ & $(1000,0,0)$ \\
\hline$f_{0}$ & $(0,0,0)$ & $(500,650,0)$ & $(500,-650,0)$ \\
\hline
\end{tabular}

(note: Fish1 is leader, so its $(\Delta x, \Delta z, \Delta \alpha)$ is $(0,0,0)$, the leader's initial coordinates to take $(-900,0,0)$ in this experiment)

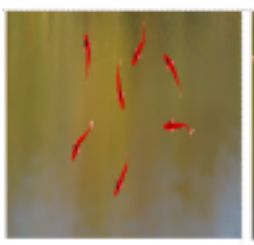

(a)

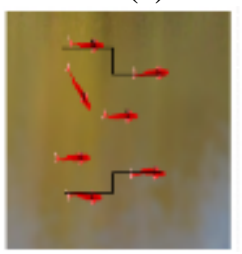

(g)

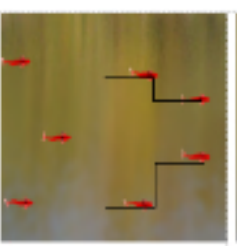

(b)

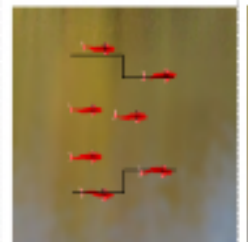

(h)

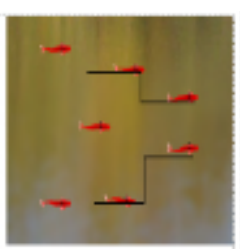

(c)

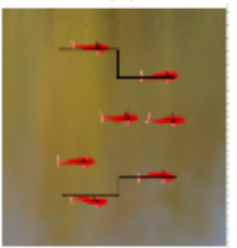

(i)

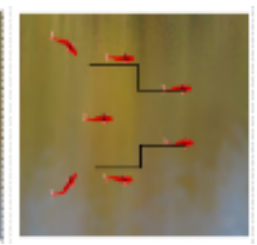

(d)

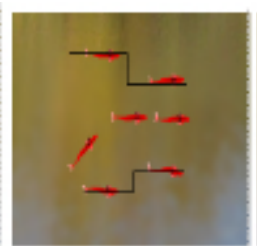

(j)

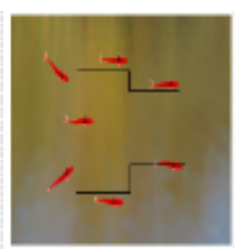

(e)

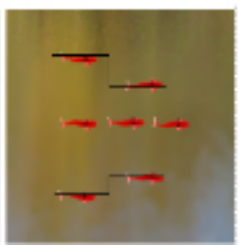

(k)

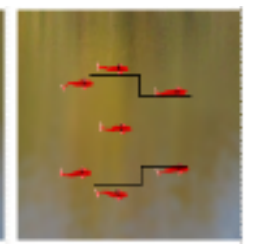

(f)

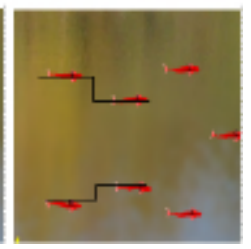

(1)

Fig. 6 Simulation of formations switching process.

First of all, the robot fishes are randomly distributed among platform, as shown in Fig. 6 (a); To initialize the robot fishes positions: create an obstacles environment and forming the initial queue (big triangular), as shown in Fig. 6(b); Keep the initial queue to move toward the target area, as shown in Fig. 6(c); Reached the barrier area consisting of fish8, and fish9, formation switch into a small triangular, as shown in Fig. 6(d) (e) (f); Maintain a small triangle formation moving on, 
reaching the barrier area formed by robot fishes numbered 6 and 7 , formation switching to a straight line, as shown in Fig. 6 (g) (h) (i) (j) (k);

When fishes swim out of hazards, and restore the initial formation, as shown in Fig. 6 (1). Now, all formations' transformation are finished successfully.

\section{Conclusion and Outlook}

This article has done a deeper level of research and experimentation in forming, maintenance and switching of formation under the leader-follower control strategy. Simulation results confirm the feasibility and effectiveness of the design idea. This method applies to the situations that has known location of obstacles. Its calculation is simple, flexible and good overall. But in many unknown deep-sea environments, they will be continued to judge and research on these problems: choosing of formation, conditions of switching, optimization of transformation efficiency and so on. They will be further explored at the later stage of research.

\section{Acknowledgements}

This work was financially supported by Innovative Research Team of the department of Sichuan Province (15TD0050) and the Fundamental Research Funds for Central University, Southwest University for Nationalities (13NZYQN04).

\section{References}

[1] L. M. Ren, W. D. Wang, Z. J. Du, Key Technologies and development of formation control of mobile robots, CAAI Trans. Intell. Syst. 8(5) (2013) 381-394.

[2] P. K. C. Wang, Navigation strategies for multiple autonomous mobile robots moving in formation, J. Robot. Syst. 8(2) (1991) 177-195.

[3] R. M. Kuppan Chetty, M. Singaperumal, T. Nagara-Jan, Behavior based multi-robot formations with active obstacle avoidance based on switching control strategy, Adv. Mater. Res.433-440 (2012) 6630-6635.

[4] D. H. Ren, G. Z. Lu, Thinking in formation control, Contr. Decis. 20(6) (2005) 601-606.

[5] K. Y. Wu, W. Huo, Indirect adaptive fuzzy dynamical formation control of uncertain mobile robots, Contr. Decis. 25(12) (2010) 1699-1781. 